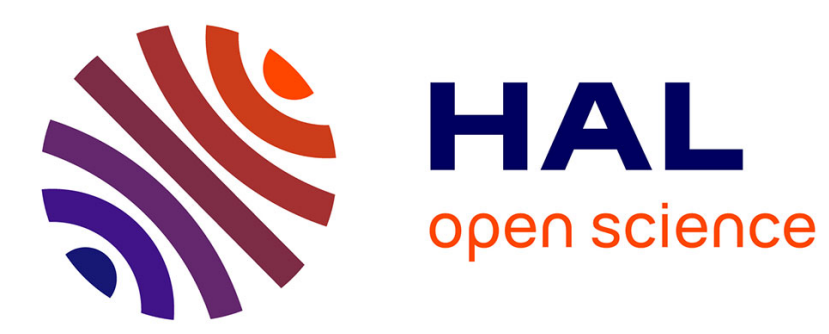

\title{
The MIRS computer package for modeling the rovibrational spectra of polyatomic molecules
}

\author{
Andrei Nikitin, Jean-Paul Champion, Vladimir G. Tyuterev
}

\section{To cite this version:}

Andrei Nikitin, Jean-Paul Champion, Vladimir G. Tyuterev. The MIRS computer package for modeling the rovibrational spectra of polyatomic molecules. Journal of Quantitative Spectroscopy and Radiative Transfer, 2003, 82, pp.239-249. 10.1016/S0022-4073(03)00156-0 . hal-00587158

\section{HAL Id: hal-00587158 https://hal.science/hal-00587158}

Submitted on 19 Apr 2011

HAL is a multi-disciplinary open access archive for the deposit and dissemination of scientific research documents, whether they are published or not. The documents may come from teaching and research institutions in France or abroad, or from public or private research centers.
L'archive ouverte pluridisciplinaire HAL, est destinée au dépôt et à la diffusion de documents scientifiques de niveau recherche, publiés ou non, émanant des établissements d'enseignement et de recherche français ou étrangers, des laboratoires publics ou privés. 


\title{
The MIRS computer package for modeling the rovibrational spectra of polyatomic molecules
}

\author{
A. Nikitin ${ }^{a}$, J. P. Champion ${ }^{b}$ and Vl. G. Tyuterev ${ }^{c}$ \\ ${ }^{a}$ Laboratory of Theoretical Spectroscopy, Institute of Atmospheric Optics, Russian Academy of Sciences, 634055, \\ Tomsk, Russia. \\ ${ }^{b}$ Laboratoire de Physique (U.M.R. C.N.R.S. 5027), Université de Bourgogne, 9 Avenue A. Savary, B.P. 47870, 21078, \\ Dijon, France. \\ ${ }^{c}$ Laboratoire de Spectroscopie Moléculaire Atmosphérique (U.M.R. C.N.R.S. 6089), Université de Reims, B.P. 1039, \\ 51623, Reims, France.

\begin{abstract}
The MIRS spectroscopic software for the modeling of ro-vibrational spectra of polyatomic molecules is presented. It is designed for the global treatment of complex band systems of molecules to take full account of symmetry properties. It includes efficient algorithms based on the irreducible tensor formalism. Predictions and simultaneous data fitting (positions and intensities) are implemented as well as advanced options related to group theory algebra. Illustrative examples on $\mathrm{CH}_{3} \mathrm{D}, \mathrm{CH}_{4}, \mathrm{CH}_{3} \mathrm{Cl}$ and $\mathrm{PH}_{3}$ are reported and the present status of data available is given. It is written in $\mathrm{C}++$ for standard $\mathrm{PC}$ computer operating under Windows. The full package including on-line documentation and recent data is freely available at the URL: http://icb.u-bourgogne.fr/OMR/SMA/SHTDS/MIRS.html.
\end{abstract}

\section{Contents}

1 Introduction

2 Modeling principles $\mathbf{2}$

2.1 Unified irreducible tensor method . . . . . . . . . . . . . . . . . . . . . . . .

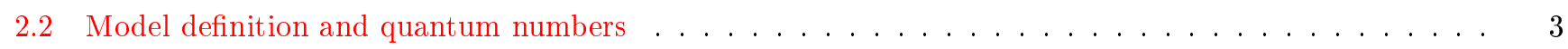

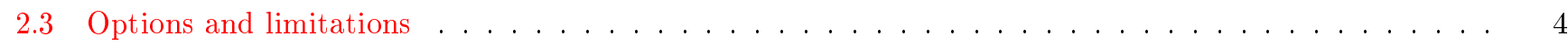

3 Basic applications $\mathbf{4}$

3.1 Prediction of energy levels and transitions . . . . . . . . . . . . . . . . . . . . 4

3.2 Present status of data available . . . . . . . . . . . . . . . . . . . . . . . .

4 Advanced applications $\mathbf{5}$

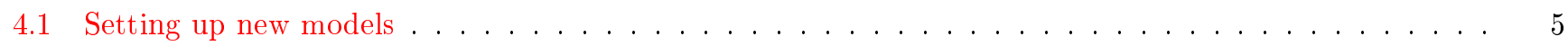

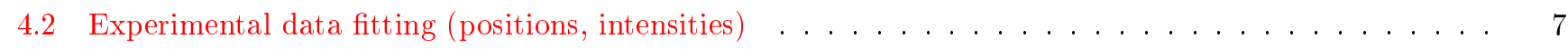

4.3 Advanced features for spectroscopic non-linear least-squares . . . . . . . . . . . . . . . . 7

4.4 Group theory algebra - commutator calculation . . . . . . . . . . . . . . . . . 7

5 Getting and installing $\quad 7$

6 Conclusion and perspectives 8

\begin{tabular}{ll} 
References & 8 \\
\hline
\end{tabular} 


\section{Introduction}

Spectroscopic investigations of polyatomic molecules play a role of primordial importance for atmospheric applications. Nowadays, in order to model the dramatic effects of ro-vibrational perturbations on line positions and strengths revealed by modern high-resolution and high-sensitivity techniques, the analysis of complex interacting band systems (so-called polyads) becomes the norm rather than the exception. Decades ago, sophisticated models have been developed and successfully applied to spherical tops in general and methane in particular [1]. For this type of molecule no simple analytical formulation was available to describe the ro-vibrational energy levels beyond the zero order of approximation. Furthermore, strong resonances between bending and stretching vibrational modes prevent from fully perturbative approaches. These particular features explain why the modeling of spherical tops relied from the beginning on numerical methods based on specific formalisms. A full account for symmetry properties proves to be crucially important for such systems. Besides purely technical advantages, such developments promoted more general concepts suitable for a wider class of molecules. One of the main achievement was the introduction of the socalled "vibrational extrapolation scheme" allowing the construction of a unified transformed hamiltonian and related transformed transition moments successfully applied to numerous global analyses. Most of the results on $\mathrm{CH}_{4}$ and $\mathrm{CH}_{3} \mathrm{D}$ are included in the HITRAN database [2].

In the present paper, we describe recent computational developments: the Windows implementation MIRS of the theoretical model published in [3] . It is especially designed for the treatment of complex band systems of molecules having symmetry properties (spherical and symmetric tops). The algorithms are based on the effective hamiltonian theory with extensive use of the irreducible tensor formalism. Predictions and global data fitting (positions and intensities) are both implemented.

The present paper was primarily motivated by sharing the concern of the HITRAN database to give friendly access to detailed results of advanced spectra analyses of symmetric top molecules. One problem is that the irreducible tensor formalism implies a non-conventional nomenclature of the effective parameters. For a given model and order of approximation, tensorial and classical expansions already available for symmetric tops are equivalent. However, due to the high flexibility of the tensorial approach, all symmetry allowed terms, sophisticated or not, are equally easy to implement. The selection of relevant terms is solely governed by physical or statistical considerations whereas in traditional approaches it interfers at least implicitly with intuitive arbitrary criteria. As a result the conversion of tensorial terms into conventional terms is made difficult not only because of the relative algebraic complexity of tensor calculations but also and principally because several tensorial terms have not yet been systematically considered in conventional formalisms. Except for simple systems like isolated vibrational states, this prevents from global conversions of complete sets of tensorial terms into complete sets of conventional terms. The theoretical investigation of the corresponding implicitly reduced hamiltonians remains not obvious in the general case. For searchers not familiar with tensorial calculus, the friendly graphical user interface of the MIRS package includes on-line documentation, and represents a powerful and flexible tool to access spectroscopic data from a standard PC computer. For expert researchers, advanced options are included such as group theory algebra and commutator calculations needed to set up reduced forms of effective hamiltonians.

For brevity, in the present paper, only the basic features will be described in some detail. Recent results of the global analysis of the lower polyads of molecules of atmospheric interest $\left(\mathrm{CH}_{3} \mathrm{D}\right.$ and $\left.\mathrm{CH}_{3} \mathrm{Cl}\right)$ are reported to illustrate the reliability and originality of the features involved.

\section{Modeling principles}

\subsection{Unified irreducible tensor method}

As described in [1], in most ro-vibrational quantitative studies, the solution of the Schrödinger equation is based on the construction of effective hamiltonians by introducing basis functions in which the hamiltonian matrix has a blockdiagonal form with respect to appropriate vibrational subspaces. There exist many ways (more or less equivalent) of defining basis functions and operators. However, in view of limitations of the standard calculation resources presently available on PC computers, considerations of computational efficiency in setting up theoretical models are 
of importance. As for previously reported softwares [4, 5, 6, 7] vibrational operators are constructed in tensor form by recursive coupling of creation and annihilation operators associated to the normal modes of the molecule. In MIRS, a specific coupling scheme associated to a binary tree is applied directly to an arbitrary number of interacting vibrational modes and to arbitrarily high polyads. The construction of vibrational basis functions is achieved consistently from the action of creation operators on the vacuum function. This method is not only satisfying conceptually but also quite efficient for the computer calculation of matrix elements and commutators. The ro-vibrational terms are easily generated by further tensor couplings. Once the vibrational polyad scheme is defined for a given molecule belonging to a given point group, the MIRS program determines automatically all symmetry allowed terms up to the desired order of approximation. The standard Amat and Nielsen classification scheme [8] is used in which the order of terms of the type $H_{m n} \sim r^{n} J^{m}$ is $m+n-2$ [9]. For transformed transition moment, terms of the type $\mu_{m n}$ are of order $m+n-1$. In general, a complete nomenclature of ro-vibrational terms appears rather cumbersome when all tensorial indices are displayed. In many cases, most of the couplings are trivial and the following simplified notation can be used unambiguously

$$
\underbrace{\Omega(K, \kappa \Gamma)} \underbrace{\left\{n_{1} \ldots n_{v}\right\}\left\{m_{1} \ldots m_{v}\right\}\left[\Gamma_{n},\left[\Gamma_{m}\right]\right]} .
$$

In the rotational part (first group of indices), $\Omega$ is the power in angular momentum components $J_{\alpha}, K$ is the tensor rank relative to the space rotation group $0(3), \chi$ refers to the projection axis $O z$, and $\Gamma$ designates the common symmetry in the molecular point group of the rotational and vibrational operators. In the vibrational part (last group of indices), the $n_{i}^{\prime} s$ (resp. $m_{j}^{\prime} s$ ) are the powers of the creation operators $a_{i}^{+}$(resp. annihilation operators $a_{j}$ ), and optionally $\Gamma_{n}$ (resp. $\Gamma_{m}$ ) specifies the internal coupling species when non-trivial. For instance, $1\left(1,0 \mathrm{~A}_{2}\right) 002000000011$ designates a term from $H_{14}$ describing the third-order Coriolis interaction between the $v_{3}=2\left(A_{1}\right)$ and $v_{5}=v_{6}=1\left(A_{2}\right)$ states of an $X Y Z_{3}\left(\mathrm{C}_{3 v}\right)$ molecule.

\subsection{Model definition and quantum numbers}

The polyad structure reflects possible vibrational quasi-degeneracies which constitutes the key for defining effective hamiltonians and consequently the theoretical model for the calculation of spectra. For a given molecule, it is essentially governed by the number of the vibrational modes and their fundamental frequencies. All harmonic oscillator energies from zero to the maximum desired value are calculated using the fundamental frequencies. This determines a reference energy level pattern which is not actually used in accurate calculations, but serves for a preliminary polyad definition only. This allows a certain flexibility to include or exclude some states according to estimations of coupling matrix elements. First, starting from zero (Ground State = Polyad 0) upwards, MIRS defines automatically the initial polyad structure according to the reference pattern and applying a threshold criterium set by the user: all vibrational states for which the energy distances are closer than a given threshold value have to be gathered in one polyad. Consequently the energy gap between subsequent polyads should be larger than this value. Of course, the strength of a perturbation depends not only on the proximity of the zero order energy levels but also on the corresponding coupling matrix elements. It is possible, at the second step, to take into account such physical considerations indirectly by "playing" with the reference level pattern: for instance the user can artificially increase the distance between reference levels in the case of weak interactions or equivalently decrease the gap between reference levels corresponding to strongly coupled but distant states. These shifts do not introduce an error in final spectrum calculations, which use true energy values. Illustrations of such flexibility are given in subsequent sections. In the frame of this approach the polyad structure of the molecule determines an effective hamiltonian expansion which serves as a theoretical model for ro-vibrational bound state calculations for the considered electronic state. The so-called vibrational extrapolation scheme is automatically implemented through the formal polyad expansion (see [1] for details).

$$
H=H_{P_{0}}+H_{P_{1}}+H_{P_{2}}+\ldots
$$

The polyad structure also determines the construction of the effective transition moments in a similar way. It determines as well the quantum numbers used to label the levels and transitions. Strict quantum numbers are related to the usual invariants. They are: the polyad number $P$, the rotational quantum number $J$ and the ro-vibrational 
symmetry species $C$. Within each $P, J, C$ block the eigenvalues sorted according to increasing energies are numbered by the index $n$ from 1 to the dimension of the block. In addition, approximate quantum numbers are generated on the basis of the eigenvector analysis. For instance, the normal mode assignment and the $K$ value (in the case of symmetric top molecules) are assigned according to the largest contribution of the wavefunction decomposition into the zero-order basis functions. These approximate quantum numbers should correspond to quantum numbers of the zero-order harmonic-oscillator + rigid-rotor approximation under the condition that corresponding physical quantities are kept near-invariant under the effect of perturbations. The latter are usually accounted for by successive contact transformations from the initial molecular hamiltonian to the effective one (2), see [1] and [10] for more detail. In strongly perturbed situations, these approximate labels may become physically irrelevant.

\subsection{Options and limitations}

In its present form, the MIRS package contains the basic coupling coefficients to work with the $\mathrm{C}_{3 v}, \mathrm{D}_{3 h}, \mathrm{O}_{h}$ and $\mathrm{T}_{d}$ point groups as well as with $\mathrm{C}_{1}$ (no symmetry). Even though, in principle, the recursive algorithms imply no limitations in angular momentum values and coupling of elementary operators, the default coefficient tables included in the package are limited to meet standard needs. In particular the angular momentum limit is 80 . Note that for the cubic groups the $G$ coefficients are calculated numerically and are identical to those included in the STDS package $[4,5]$. In contrast with STDS, the number and symmetry species of normal modes are arbitrary. This means that, when physically justified, some normal modes can be omitted for the purpose of simplifying the model. Orders of expansion are also unlimited in principle, but limited in the package to the eighth order to keep the size of the coefficient tables within reasonable bounds. As described previously the MIRS program offers a large flexibility in defining the polyad structure on the basis of simple key values.

Finally, all symmetry allowed terms in the transformed Hamiltonian, vibrationally diagonal or not, and the transition moments (only dipolar transitions are implemented to date) are automatically generated to the desired order. Quite large sets of effective parameters can be generated by this procedure which means that limitations arise actually from theoretical rather than from program considerations.

\section{Basic applications}

The first application of the MIRS package is the diffusion of the results of spectroscopic analyses in a flexible way. As a matter of fact, from the files containing the model features and the effective parameter values, different kinds of prediction can be made by non-expert users.

\subsection{Prediction of energy levels and transitions}

Complete files of energy levels can be calculated using standard menu commands. Each record contains the energy value, the set of strict quantum numbers (see previous section), other approximate quantum numbers, depending on the symmetry point group and optional informations derived from the eigenvector coefficients to determine the approximate quantum numbers. Details on the experimental measurements fitted are also provided. The number and the rms deviations of assigned transitions are given together with the corresponding averaged observed minus calculated residual.

Similar details are provided for transitions (calculated positions and intensities) using standard options. The partition function may be derived from the calculated levels involved in the model or set to an appropriate external estimate. Several output formats including HITRAN [2] and GEISA [11] can be selected. The temperature and intensity threshold can be adjusted to meet specific needs for applications.

\subsection{Present status of data available}

The package presently available on our Web Site ${ }^{1}$ includes tutorial examples as well as complete projects related to recent analyses.

\footnotetext{
${ }^{1}$ http://icb.u-bourgogne.fr/OMR/SMA/SHTDS/MIRS.html
} 
The most simple example is the sixth-order model for the ground-state and the bending dyad of ${ }^{12} \mathrm{CH}_{4}$. In this case, the vibrational coupling scheme coincides exactly with the one used in the STDS program. It means that the effective parameter sets are identical in MIRS and STDS providing a good validation of both programs. Unfortunately, from the next polyad upwards even though the models are perfectly equivalent and include the same number of parameters at a given order of approximation, the two parameter sets are not in one to one correspondence. A similar system is illustrated with the sixth order model for the ground-state and the $\nu_{2} / \nu_{4}$ dyad of $\mathrm{PH}_{3}$.

The model for the lower two polyads (triad and nonad) of $\mathrm{CH}_{3} \mathrm{D}$ represents the most complex band systems of a symmetric top studied so far. Some 10,000 line positions and 2400 line intensities have been fitted to an accuracy close to the experimental precision (about $0.001 \mathrm{~cm}^{-1}$ for positions and $4 \%$ for intensities) using a common set of 441 effective hamiltonian parameters to describe 13 vibrational states (ground, triad, nonad) corresponding to 39 bands ( 6 fundamental, 3 overtone, 3 combination, and 27 hot bands). The intensities of the 9 bands involved in the nonad-ground state system were fitted to the second order of approximation using 83 effective dipole moment parameters.

Finally, very recent results of the global analysis of the lower polyads of ${ }^{12} \mathrm{CH}_{3}{ }^{35} \mathrm{Cl}$ and ${ }^{12} \mathrm{CH}_{3}{ }^{37} \mathrm{Cl}$ are included. A preliminary analysis of the infrared spectrum in the region from 0 to $1800 \mathrm{~cm}^{-1}$ was performed using 125 effective parameters for both isotopomers to model 12 sets of transitions ( 6 cold bands, 3 hot bands and 3 pure rotational systems). The precision on positions is of the order of $0.0002 \mathrm{~cm}^{-1}$.

\section{Advanced applications}

The above results have been obtained using the advanced tools included in MIRS for the modeling and the fit of experimental data. Various options are available and described in the on-line documentation of the downloadable package. Only the basic ideas will be reported here.

\subsection{Setting up new models}

By default, MIRS is set to built partially transformed hamiltonian matrices and transition moments according to the vibrational extrapolation scheme. This means that for a given molecule a common set of effective hamiltonian parameters is set up to fit and predict the subsequent vibrational polyads. The same principle applies for transition moment parameters. For instance a band system from the ground state to a given polyad and all the corresponding hot band systems are described by a common set of effective parameters. Such features are generated automatically by the program and controlled by a few basic modeling parameters entered through the window menu reproduced in Fig. 1.

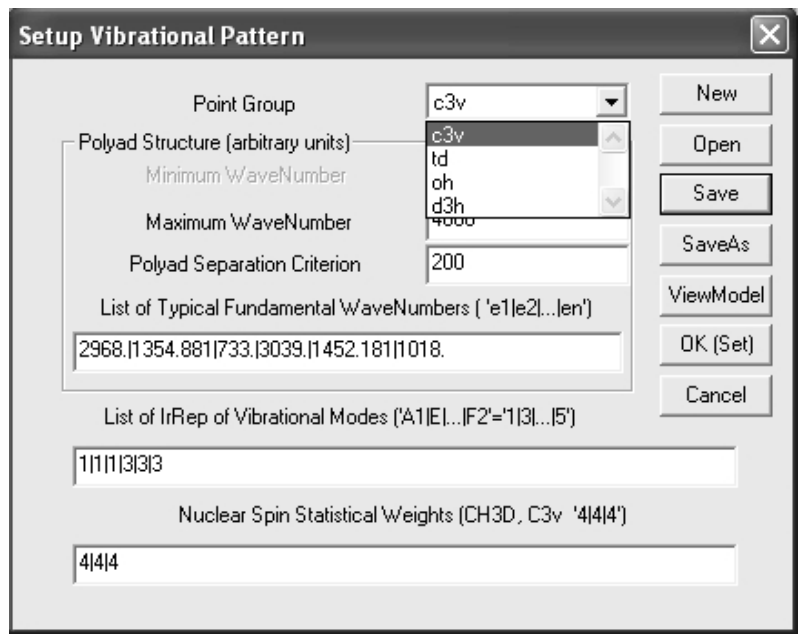

Figure 1. Graphical user interface to enter the modeling parameters: the example of CH3Cl. 
The modeling parameters are (i) the symmetry point group of the molecule, (ii) the harmonic frequencies of the desired normal modes, (iii) the corresponding symmetry species, (iv) the frequency gap used for grouping the subsequent vibrational sublevels into relevant vibrational polyads. Note that in the modeling procedure the frequencies can be entered in arbitrary units and do not need to match exactly the physical frequencies in order to provide flexibility. For example, the modeling of the $\mathrm{CH}_{3} \mathrm{D}$ molecule was performed by setting the six fundamental frequencies to 2, 2, 1, 2, 1 and 1 respectively and the frequency gap to 0.1 . This yielded the triad and nonad structure described in [12]. For $\mathrm{CH}_{3} \mathrm{Cl}$ the situation was more complex and the fundamental frequencies were set closer to the actual values: 2968, 1354, 733, 3039, 1452 and 1018, respectively with a frequency gap of 200 (Fig. 1) [13]. In all cases, the resulting polyad structure can be displayed for checking and modifying purposes.

The nuclear spin statistical weights involved in intensity calculations are also manually introduced at this stage. Once the vibrational model is set up, the polyads and the transitions to consider are entered through another window menu examplified in Fig. 2. For each polyad the order of the hamiltonian expansion and the maximum value of $J$ has to be entered. Similarly, for each type of transitions the order of the dipole moment expansion has to be specified.

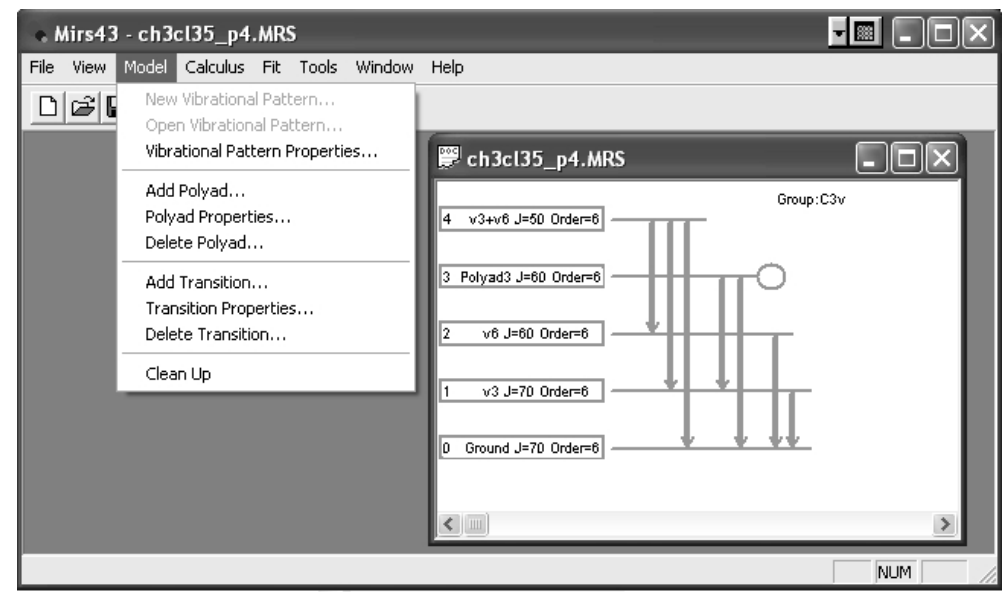

Figure 2. Graphical user interface to set up model properties. The example of CH3Cl.

At the end of the procedure the build command generates all internal files needed for subsequent calculations. The same command is used to update all files whenever needed. The other commands of the Calculus menu displayed in Fig. 3 are generally self-explanatory. More details are available from the Help command.

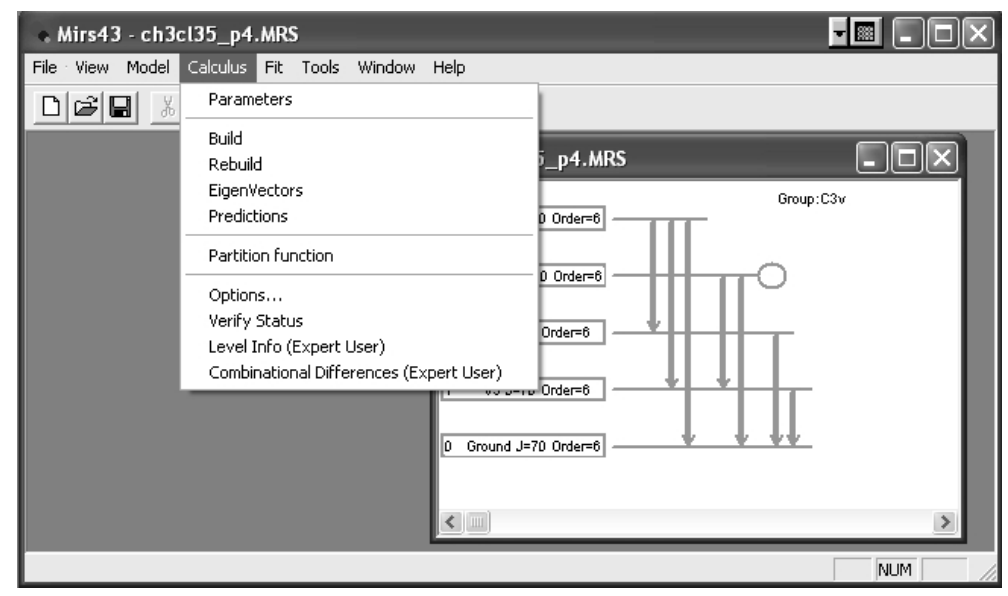

Figure 3. Graphical user interface for calculation options.

Thanks to its intrinsic flexibility MIRS can also be used to create quantum model hamiltonians not necessarily connected to real molecules. For instance, various systems involving coupled harmonic or anharmonic oscillators can be simulated numerically for further theoretical investigations. 


\subsection{Experimental data fitting (positions, intensities)}

MIRS offers standard options for non-linear least-squares fit of spectroscopic data (positions and intensities). In coherence with the vibrational extrapolation scheme well suited for global analyses, the program is set to fit simultaneously all transitions involved in a given project. As far as possible the physical quantities fitted are directly the measured transition frequencies and intensities. It is also possible to include indirect measurements such as "observed" energy levels. Note that, due to their high-sensitivity and high-resolution, modern spectroscopic techniques provide wealth of transitions so that many energy levels are involved in several observed transitions. In our approach, the direct simultaneous fit of such transitions indirectly accounts for the so-called combination differences (see Fig. 3 of [14]).

The fit of intensities is also achieved using a non-linear least-squares procedure with an adequate weighting already described in $[4,5]$. Note that in our approach a rigorous coherence is de facto applied between intensity and position fits.

The development of new algorithms and softwares was in fact justified by the increasing complexity of the problems to solve. In particular, the large number of adjustable parameters involved in global analyses required specific features in fitting procedures.

\subsection{Advanced features for spectroscopic non-linear least-squares}

In complex situations, it is difficult to select a priori the relevant parameters among a large number of candidates at a given order of approximation. Statistical correlations arise not only from possible deficiencies of the coverage by experimental data but also from essential colinearities in effective hamiltonians [15, 14, 16]. To help selecting adjustable parameters at any stage of the iterative assignment procedure special options of MIRS allow to run series of iterations with automatic introduction of new parameters or elimination of statistically unsignificant parameters among pre-selected terms specified by the user.

Another difficulty is encountered when approximate quantum numbers are not physically meaningful and stable. As mentioned previously, in MIRS, the assignment of observed to calculated data is based on the strict quantum numbers $P, J$ and $C$ (see previous section) and the numbering index $n$ within each $P, J, C$ block. In some cases close eigenvalues may permute their numbering indices from one iteration to the next causing undesirable assignment jumps. MIRS allows to remove temporarily from the fit the assigned levels capable of such behaviour by detecting anomalous variations of the scalar product of the corresponding eigenvectors from one iteration to the next.

The fit of intensities is also subject to specific problems. In particular the signs of the transition parameters cannot be completely determined from the fit of data. Some of the relative signs may be correlated with those of the interaction hamiltonian parameters. To help in such situations, MIRS can be set to run series of iterations to test the relative signs of pre-selected terms.

However, despite all the above advanced options, it should be emphasized that in any case a purely automatic selection of adjustable terms for positions or intensities is hopeless. All procedures have to rely on physical considerations.

\subsection{Group theory algebra-commutator calculation}

MIRS can also be used by expert users to generate various types of coupling coefficients as well as commutators of tensor operators in algebraic or numerical formats. Such tools are useful for the theoretical investigation of the fundamental properties of effective hamiltonians and the definition of reduced forms of effective hamiltonians [15, 13, 12]. The present version of the package includes typical sample outputs related to S-generators of contact transformations involved in the effective hamiltonian for the triad of $\mathrm{CH}_{3} \mathrm{D}$.

\section{$5 \quad$ Getting and installing}

The MIRS package can be downloaded freely from our Web site ${ }^{2}$. It consists of a self-extractive archive containing various tutorial examples and complete projects on $\mathrm{CH}_{3} \mathrm{D}$ and $\mathrm{CH}_{3} \mathrm{Cl}$. MIRS written in $\mathrm{C}++$ includes all necessary

\footnotetext{
${ }^{2}$ http://icb.u-bourgogne.fr/OMR/SMA/SHTDS/MIRS.html
} 
executable files for Windows 95 and higher. The CPU and disk space requirements obviously depend on the complexity of the project. The package itself requires a minimum of $15 \mathrm{MB}$ of free disk space. The tables of coupling coefficients occupy up to $150 \mathrm{MB}$ when the highest $J$ value (80) is involved. All examples and projects included in the package can be executed using a computer with $256 \mathrm{MB}$ RAM and $500 \mathrm{MB}$ of free disk space. Typical execution times using a processor at $2 \mathrm{GHz}$ are about 10 min to build the $\mathrm{CH}_{3} \mathrm{D}$ project (all matrix element and prediction files) and less than 15 min to build each of the $\mathrm{CH}_{3} \mathrm{Cl}$ projects.

Standard features and options are directly accessible from the tutorial projects in which all necessary modeling files are included. The Help menu can be accessed at any stage of the project building and subsequent calculations. Refer to the Web site for complete installation and running instructions.

\section{Conclusion and perspectives}

The main features of the MIRS software for modeling the ro-vibrational spectra of polyatomic molecules have been described. They correspond to the version of the package presently downloadable from the Web site http://icb.ubourgogne.fr/OMR/SMA/SHTDS/MIRS.html. It includes all executable files, tutorial examples as well as complete results of recent analyses of infrared spectra of $\mathrm{CH}_{3} \mathrm{D}$ already transferred into the HITRAN database and $\mathrm{CH}_{3} \mathrm{Cl}$ about to be submitted. The MIRS package can be used at different levels of expertise. Basically it can be used simply to generate lists of calculated energy levels and transitions from effective parameters. It is especially designed for the modeling and the global analysis of complex vibration rotation spectra (dipolar transition). It may also be used to simulate quantum model hamiltonians. Future developments and results of analyses in progress (higher vibrational polyads of $\mathrm{CH}_{3} \mathrm{Cl}$ ) will be made available from the above mentioned Web Site.

\section{Acknowledgements}

Part of the work was supported by the Institut National des Sciences de l'Univers under the frame of the Programme National de Chimie Atmosphérique and by CNRS (France)-RFBR (Russia) under the frame of the Programme Intègré de Coopération Scientifique No. 1572.

\section{References}

[1] J. Champion, M. Loete, G. Pierre, Spherical top spectra, in: K. Rao, A. Weber (Eds.), Spectroscopy of the Earth's atmosphere and interstellar medium, Academic Press Inc., Columbus, 1992, pp. 339-422. 2, 3, 4

[2] L. R. Brown, D. C. Benner, J. P. Champion, V. M. Devi, L. Fejard, R. R. Gamache, T. Gabard, J. C. Hilico, B. Lavorel, M. Loete, G. C. Mellau, A. Nikitin, A. S. Pine, A. Predoi-Cross, C. P. Rinsland, O. Robert, R. L. Sams, M. A. H. Smith, S. A. Tashkun, V. G. Tyuterev, Methane line parameters in hitran, Journal of Quantitative Spectroscopy \& Radiative Transfer 82 (1-4) (2003) 219-238. doi:10.1016/S0022-4073(03)00155-9. 2, 4

[3] A. Nikitin, J. P. Champion, V. G. Tyuterev, Improved algorithms for the modeling of vibrational polyads of polyatomic molecules: Application to t-d, o-h, and c-3 upsilon molecules, Journal of Molecular Spectroscopy 182 (1) (1997) 72-84. 2

[4] C. Wenger, J. P. Champion, Spherical top data system (stds) software for the simulation of spherical top spectra, Journal of Quantitative Spectroscopy \& Radiative Transfer 59 (3-5) (1998) 471-480. 3, 4, 7

[5] C. Wenger, V. Boudon, J. P. Champion, G. Pierre, Highly-spherical top data system (htds) software for spectrum simulation of octahedral xy6 molecules, Journal of Quantitative Spectroscopy \& Radiative Transfer 66 (1) (2000) 1-16. $3,4,7$

[6] C. Wenger, M. Rotger, V. Boudon, C4(v) top data system (c4(v) tds) software for infrared spectrum simulation of xy(5)z symmetric molecules, Journal of Quantitative Spectroscopy \& Radiative Transfer 74 (5) (2002) 621-636. 3 
[7] M. Rotger, V. Boudon, M. Loete, Spectroscopy of $x y(2) z(2)(c 2 v)$ molecules: A tensorial formalism adapted to the $\mathrm{o}(3)$ superset of $\mathrm{t}-\mathrm{d}$ superset of $\mathrm{c}-2 \mathrm{v}$ chain. application to the ground state of so2f2, Journal of Molecular Spectroscopy 216 (2) (2002) 297-307. doi:10.1006/jmsp.2002.8635. 3

[8] G. Amat, H. H. Nielsen, G. Tarrago, Rotation - vibration of polyatomic molecules, Dekker, New York, 1971.3

[9] M. Aliev, J. Watson, Higher-order effects in the vibration-rotation spectra of semirigid molecules, in: K.N.Rao (Ed.), Molecular Spectroscopy: Modern Research, Vol. III, Academic Press, New York, 1985, pp. 1 -67. 3

[10] B. Zhilinskii, V. Perevalov, V. Tyuterev, Method of irreducible tensorial operators in the theory of molecular spectra. (in Russian), Nauka, Novosibirsk, 1987. 4

[11] N. Jacquinet-Husson, E. Arie, J. Ballard, A. Barbe, G. Bjoraker, B. Bonnet, L. R. Brown, C. Camy-Peyret, J. P. Champion, A. Chedin, A. Chursin, C. Clerbaux, G. Duxbury, J. M. Flaud, N. Fourrie, A. Fayt, G. Graner, R. Gamache, A. Goldman, V. Golovko, G. Guelachvili, J. M. Hartmann, J. C. Hilico, J. Hillman, G. Lefevre, E. Lellouch, S. N. Mikhailenko, O. V. Naumenko, V. Nemtchinov, D. A. Newnham, A. Nikitin, J. Orphal, A. Perrin, D. C. Reuter, C. P. Rinsland, L. Rosenmann, L. S. Rothman, N. A. Scott, J. Selby, L. N. Sinitsa, J. M. Sirota, A. M. Smith, K. M. Smith, V. G. Tyuterev, R. H. Tipping, S. Urban, P. Varanasi, M. Weber, The 1997 spectroscopic geisa databank, Journal of Quantitative Spectroscopy \& Radiative Transfer 62 (2) (1999) $205-254$. 4

[12] A. Nikitin, L. R. Brown, L. Fejard, J. P. Champion, V. G. Tyuterev, Analysis of the ch3d nonad from 2000 to $3300 \mathrm{~cm}(-1)$, Journal Of Molecular Spectroscopy 216 (2) (2002) 225-251. 6, 7

[13] A. Nikitin, L. Fejard, J. P. Champion, H. Burger, M. Litz, J. M. Colmont, B. Bakri, New measurements and global analysis of chloromethane in the region from 0 to $1800 \mathrm{~cm}(-1)$, Journal of Molecular Spectroscopy 221 (2) (2003) 199-212. doi:10.1016/S0022-2852(03)00221-2.6, 7

[14] V. G. TYUTEREV, J. P. CHAMPION, G. PIERRE, Reduced effective-hamiltonians for degenerate excited vibrational-states of tetrahedral molecules - application to 2-nu-2,nu-2+nu-4 and 2-nu-4 of ch4, Molecular Physics 71 (5) (1990) 995-1020. 7

[15] V. G. TYUTEREV, J. P. CHAMPION, G. PIERRE, V. I. PEREVALOV, Parameters of reduced hamiltonian and invariant parameters of interacting e and f2 fundamentals of tetrahedral molecules - nu- 2 and nu- 4 bands of (ch-2)-c-4 and(sih-28)-s-4, Journal of Molecular Spectroscopy 120 (1) (1986) 49-78. 7

[16] E. Lobodenko, O. Sulakshina, V. Perevalov, V. Tyuterev, Reduced effective hamiltonian for coriolis-interacting nn and nt fundamentals of c3v molecules, J. Mol. Spectrosc. 126 (1987) 159-170. 7 